\title{
ADMINISTRATION OF 4-HYDROXY-3,5-DI-TERTBUTYL CINNAMIC ACID RESTORES MITOCHONDRIAL FUNCTION IN RABBITS WITH CEREBRAL ISCHEMIA
}

\author{
Dmitriy Pozdnyakov ${ }^{1}$, Zara Hadzhieva $^{2}$ and Anastasiya Pozdnyakova ${ }^{2}$
}

${ }^{1}$ Pyatigorsk Medical and Pharmaceutical Institute, Department of Pharmacology with course in Clinical Pharmacology, Pyatigorsk, Russia ${ }^{2}$ Pyatigorsk Medical and Pharmaceutical Institute, Department of Pharmaceutical Technology with course in Biotechnology, Pyatigorsk, Russia

\section{Corresponding author:}

\section{Dmitriy Igorevich Pozdnyakov,}

Pyatigorsk Medical and Pharmaceutical Institute Department of Pharmacology with course in Clinical Pharmacology

357532 Pyatigorsk, Russia

E-mail: pozdniackow.dmitry@yandex.ru

\section{sciendo}

UDK: 616.831-005.4:577.1 576.311 .347

Ser J Exp Clin Res 2022; 23(2):155-165

DOI: $10.2478 /$ sjecr-2019-0075

\begin{abstract}
The aim of the study is to evaluate the effect of 4-hydroxy-3,5-ditertbutyl cinnamic acid on the change in mitochondrial function under conditions of experimental cerebral ischemia in rabbits. The study was performed on 48 male rabbits, which were used for modeling permanent cerebral ischemia by occlusion of the common carotid arteries. The test compound was administered before modeling ischemia for 14 days and after the occurrence of reproducing ischemia, in a similar time interval. After that, neurological deficit and the parameters of mitochondrial respiration, the intensity of anaerobic processes, the latent opening time of the mitochondrial permeability transition pore, the value of the mitochondrial membrane potential and the concentration of caspase - 3 were determined. The administration of $100 \mathrm{mg} / \mathrm{kg}$ of 4-hydroxy-3,5-di-tertbutyl cinnamic acid into the animals reduced neurological deficit and restored the mitochondrial membrane potential. Prophylactic administration of 4-hydroxy-3,5-ditertbutyl cinnamic acid, contributed to an increase in ATPgenerating ability, the maximum level of respiration and respiratory capacity by 4.1 times $(p<0.01), 4.8$ times $(p<0.01)$ and 4.3 times $(p<0.01)$, respectively. With therapeutic administration, these indicators increased by 11 times $(p<0.01), 12.2$ times $(p<0.01)$ and 8.6 times $(p<0.01)$, respectively. Also, both the prophylactic and therapeutic use of 4-hydroxy-3,5-di-tret-butyl cinnamic acid normalized aerobic/anaerobic metabolism, as well as reduced the concentration of caspase-3. Based on the obtained data, significant cerebroprotective properties of 4-hydroxy-3,5di-tertbutyl cinnamic acid can be assumed. Moreover, the potential mechanism of action of this compound may be mediated by the normalization of mitochondrial function.
\end{abstract}

Keywords: cerebral ischemia, cinnamic acid derivatives, mitochondrial dys-function. 


\section{INTRODUCTION}

Stroke is the most common cerebrovascular accident, as well as one of the leading causes of death and primary disability in both economically developed and developing countries (1). The typology of stroke includes hemorrhagic and ischemic subtypes, and the latter accounts for about $70-80 \%$ of all cases of stroke (2). In recent years, significant progress has been made in understanding the pathophysiology of ischemic stroke, which has significantly optimized therapeutic approaches to the treatment of this condition (3). However, relevant statistical data, as well as prognostic calculations by specialists of the World Health Organization, show a progressive increase in mortality from cerebrovascular diseases in the acute phase. In low-income countries, stroke ranks fifth among the causes of death, at the same time with an increase in the well-being of the population, i.e. in middle-low, middle-high and high-income countries, ischemic brain damage already takes the second place in the structure of total mortality. Moreover, as a rule, ischemic stroke occurs in people of working age, which negatively affects the economic activity of a number of countries (3). Etiopathogenetic stroke is characterized by cerebral vessel occlusion with the formation of a necrotic zone - a zone of cerebral infarction ("ischemic shadow"), surrounded by tissue with reduced blood flow and metabolic activity ("ischemic penumbra"), which, if an unfavorable outcome occurs, can be involved in the formation of a brain tissue necrosis zone (5). In the "ischemic penumbra", there is an increase in inflammation, oxidative stress, glutamate excitotoxicity and intracellular calcium concentration, as well as a decrease in ATP synthesis and activation of apoptosis, and activation of anaerobic processes with hyperlactatemia (6). In the activation of the above processes, a special role is given to the mitochondria of neurons. Mitochondria are cellular organelles that mainly perform an energy-producing function (macroergic substrates in the form of ATP): however, the functional activity of mitochondria is also realized in the regulation of the cell redox state and apoptosis (7). Active forms of oxygen are by-products of normal mitochondrial metabolism, which play a significant negative role in the pathogenesis of ischemic stroke, initiating the processes of cellular and subcellular lipoperoxidation, as well as activating calcium influx, increasing its intracellular concentration to a toxic level (8). Mitochondrial-dependent apoptosis, as a rule, is activated upon reaching the minimum ATP level acceptable for normal cell functioning and includes caspasedependent and caspase-independent cascade reactions (9). The effector system of caspase-dependent apoptosis is caspase-3, which acts as an enzyme, a DNA sequencer (10). Caspase-independent apoptosis is activated by decreasing mitochondrial membrane potential, resulting in opening of the mitochondrial permeability transition pore with cytochrome $\mathrm{C}$ releasing, activation of the apoptosis-inducing factor and formation of apoptosome (11). As a result of the described pathogenetic mechanisms, intensification of neuronal death in the "ischemic penumbra" and an increase in the zone of cerebral infarction are noted, which in turn contribute to the clinical symptoms of ischemic stroke: cognitive dysfunction, neurological deficit, sensorimotor disorders, paresthesia, and depression (12). In this regard, it can be assumed that a targeted effect on mitochondria can reduce the degree of brain damage in conditions of ischemic stroke. In previous studies, potential cerebroprotective properties of 4hydroxy-3,5-di-tertbutyl cinnamic acid have been identified, which may also be associated with the restoration of mitochondrial function by stabilizing the activity of cytochrome C oxidase and ATP synthetase (mitochondrial complex IV and $\mathrm{V}$ respectively) (13). In this respect, the aim of this study was to evaluate the effect of 4-hydroxy-3,5-di-tertbutyl cinnamic acid on the change of functional activity of the brain mitochondria of rabbits in conditions of cerebral ischemia.

\section{MATERIALS AND METHODS}

\section{Experimental animals}

As a biological model, this study used 48 male Soviet Chinchilla rabbits weighing $2300-2500 \mathrm{~g}$. The animals were kept in standard living system laboratory conditions at the Pyatigorsk Medical and Pharmaceutical Institute with a natural change in the daily cycle (12 hours a day, 12 hours a night), relative humidity $60 \pm 5 \%$ and ambient temperature $20 \pm 30^{\circ} \mathrm{C}$. The rabbits were housed in individual cages with free access to food and water. The animals were removed from the experiment by cervical dislocation under chloral hydrate anesthesia (chloral hydrate $350 \mathrm{mg} / \mathrm{kg}$, intraperitoneally). The keeping and manipulation of animals was in accordance with generally accepted standards of experimental ethics (Directive 2010/63/EU of the European Parliament and of the Council on the protection of animals used for scientific purposes, adopted on September 22, 2010).

\section{Test objects}

In this study, the test object was 4-hydroxy-3,5-di-tertbutyl cinnamic acid (Fig. 1). The studied substance was obtained at the Department of Organic Chemistry of the Pyatigorsk Medical and Pharmaceutical Institute under the supervision of professor E.T. Oganesyan. The authenticity of the compound was confirmed by UV, IR and NMR spectroscopy. Ethylmethylhydroxypyridine succinate (Mexidol, FARMASOFT, Russia) at a dose of $100 \mathrm{mg} / \mathrm{kg}$ was used as a reference drug (14).

Figure 1. The structure of 4-hydroxy-3,5-di-tertbutyl cinnamic acid.

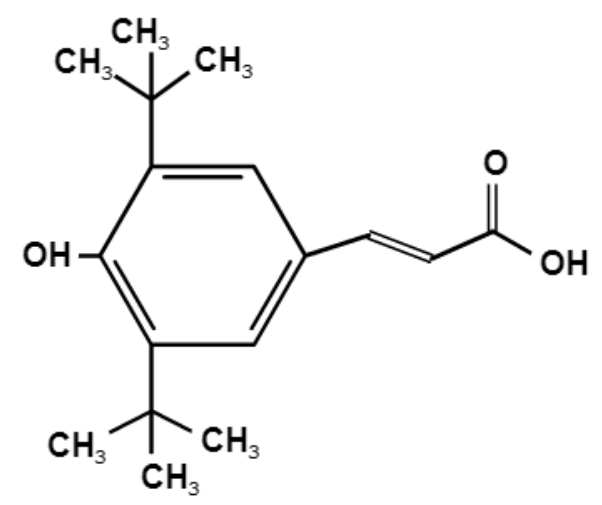




\section{Study design}

The study was approved by the local ethics committee (protocol No. 18 of June 25,2019). The study was realized in 2 steps. In the first step, the pharmacological efficacy of the test compound and the reference drug were evaluated with prophylactic administration. In the second step, the test object and reference drug were introduced after modeling cerebral ischemia in order to assess the therapeutic effect. In the course of the experiment, at each of the steps, six equal experimental groups of animals were formed $(n=4$ in each group). The first group included sham-operated animals (SO), to which all successive surgical procedures were applied except ligation of the common carotid arteries. The second group was the negative control group (NC); it included animals deprived of pharmacological support (this group of animals was administered isotonic sodium chloride solution).
Groups No. 3-5 were given 4-hydroxy-3,5-di-tertbutyl cinnamic acid (laboratory code ATACL) at doses of $50 \mathrm{mg} / \mathrm{kg}$, $100 \mathrm{mg} / \mathrm{kg}$, and $150 \mathrm{mg} / \mathrm{kg}$, respectively Animals of the sixth group were treated with the reference drug, Mexidol, at a dose of $100 \mathrm{mg} / \mathrm{kg}$. In the first step of the study, the ATACL compound and the reference drug were administered per os for 14 days before the brain ischemia modeling. In the second stage of the experimental work, the ATACL substance and the reference drug were administered intragastrically after reproducing ischemic brain damage for 14 days. Animals were removed from the experiment by cervical dislocation with decapitation in the first step of the experiment, 24 hours after the reproduction of ischemia. In the second step of the experiment, the animals were removed after 14 days of administration of the test compound and the reference drug. The study design is presented in Fig. 2.

Figure 2. Study design

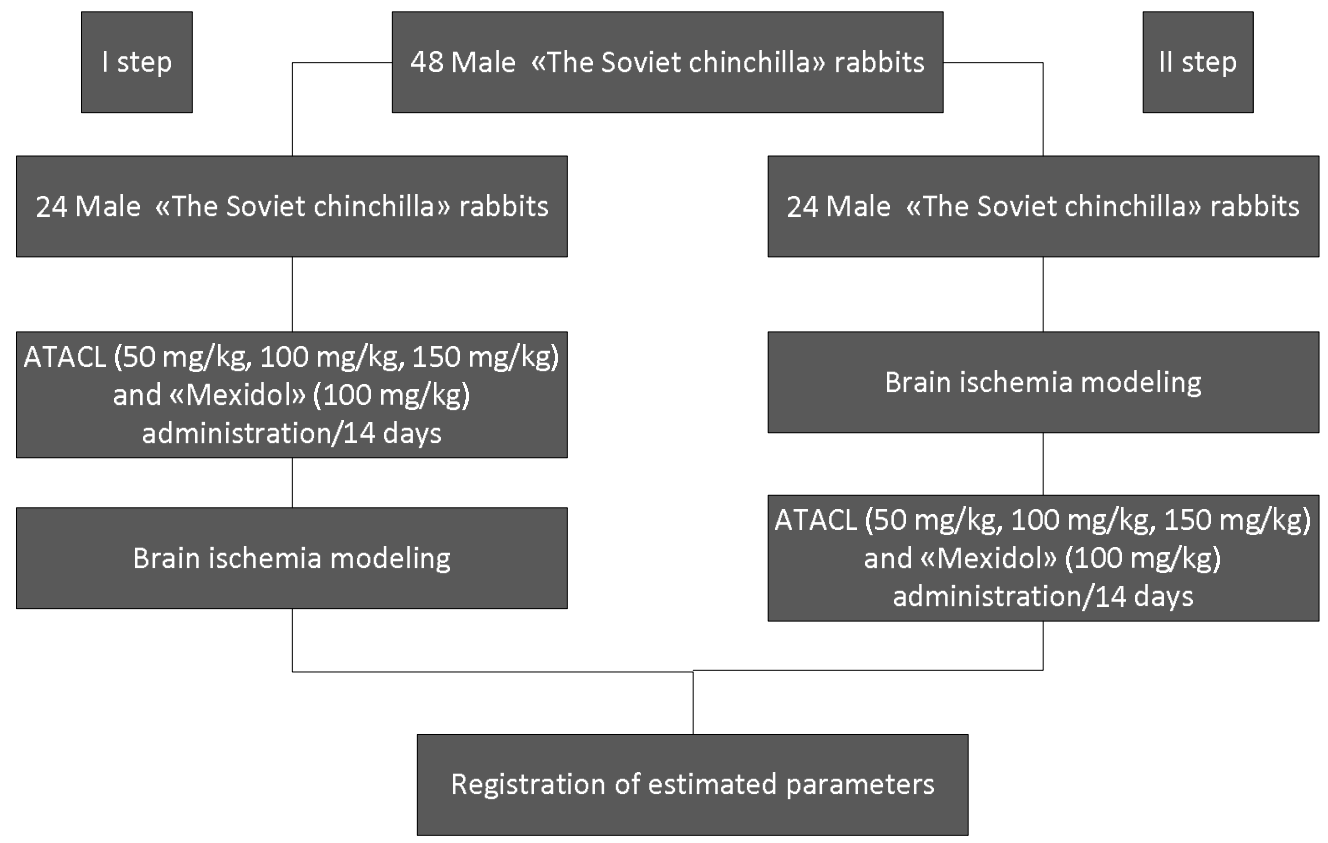

\section{Cerebral ischemia model}

Cerebral ischemia in rabbits was modeled by ligation of the common carotid arteries (subtotal permanent brain ischemia). In anesthetized animals (acepromazine $1 \%$ solution, $0.05 \mathrm{ml} / \mathrm{kg}$, intramuscularly + chloral hydrate $350 \mathrm{mg} / \mathrm{kg}$, intraperitoneally + atropine sulfate $0.1 \%, 0.1 \mathrm{ml}$, subcutaneously), the fell on the lower side of the neck was removed. Then an incision was made and soft tissues and muscles were dissected, after which the left and right common carotid arteries were visually detected. The arteries were isolated, cleaned of adjacent tissues and nerve fibers, after which a silk thread was inserted under the artery, which was used to bind the artery, while the termination of blood flow was visually noted above the ligation site. The tissue topography was then restored, and the wound was sutured and treated by antiseptic (Benzyldimethyl (3-myristoilamine) propyl) ammonium chloride monohydrate); $0.01 \%$ solution).

\section{Neurological deficit determination}

The degree of development of neurological deficit was evaluated according to the McGraw scale (1976) according to the parameters presented in Table 1 .

Table 1. McGraw neurological deficiency scale (1976)

\begin{tabular}{|l|l|}
\hline Symptoms / parameters & Neurological deficit score \\
\hline Inertness & 0.5 \\
\hline Tremor & 1 \\
\hline
\end{tabular}




\begin{tabular}{|l|l|}
\hline Symptoms / parameters & Neurological deficit score \\
\hline One-side hemiptosis & 1 \\
\hline Bi-side hemiptosis & 1.5 \\
\hline Limb weakness & 1.5 \\
\hline One-side ptosis & 1.5 \\
\hline Bi-side ptosis & 1.5 \\
\hline Circular motion & 2.0 \\
\hline Limbs paresis (1-4) & $2-5$ \\
\hline Limb paralysis (1-4) & $3-6$ \\
\hline Coma & 7.0 \\
\hline Death & 10.0 \\
\hline
\end{tabular}

The total score of 0.5-2.0 corresponded to a mild degree of neurological deficit; the score of 2.5-5.0 presented moderate severity; the score of 5.5-10 presented severe neurological deficit, while the degree of neurological deficit was determined by the sum of the relevant points (15).

\section{Biomaterial sample preparation}

Brain was used as biomaterial. The animals were decapitated under anesthesia and the organs were collected and divided into two parts, after which the first part of the biomaterial was homogenized in a Potter mechanical homogenizer in a selection medium (1 mmol EDTA, $215 \mathrm{mmol} \mathrm{man-}$ nitol, $75 \mathrm{mmol}$ sucrose, $0.1 \%$ BSA solution, $20 \mathrm{mmol}$ HEPES, with a $\mathrm{pH}$ 7.2). The cell population was obtained by differential centrifugation, for which the obtained biogenic homogenate was centrifuged in the mode of $1400 \mathrm{~g}$ by $3 \mathrm{~min}$ at $40{ }^{\circ} \mathrm{C}$, after which the supernatant was transferred to $2 \mathrm{ml}$ tubes. Next, the resulting supernatant was centrifuged at $13000 \mathrm{~g}$ for $10 \mathrm{~min}$ and the supernatant (culture contains native mitochondria) was removed for respirometric analysis (16). The second part of the brain was homogenized in PBS with a pH 7.4 in a ratio of 1:7 and centrifuged in the mode of $10000 \mathrm{~g}$ for $5 \mathrm{~min}$, then the resulting supernatant was taken for ELISA.

\section{Respirometric analysis}

Analysis of mitochondrial respiratory function was carried out by the method of respirometry using the AKPM101L laboratory respirometer system (Alfa Bassens, Russia). The mitochondrial respiratory function was assessed by the change in oxygen consumption in the medium against the introduction of mitochondrial respiratory uncouplers. In the last stage, there was a sequential addition of oligomycin -1 $\mu \mathrm{g} / \mathrm{ml}, 4$ - (trifluoromethoxy) phenyl) hydrazono) malononitrile (FCCP-1 $\mu \mathrm{M}$ ), rotenone - $1 \mu \mathrm{M}$, and sodium azide - 20 mmol. Glucose was used as an oxidation substrate- at a dose of $15 \mathrm{mmol}$ The overall assessment of mitochondrial function was determined by the level of oxygen consumption in the medium after sequential addition of oligomycin, FCCP and rotenone to the medium. The ATP-generating ability was determined by the difference in oxygen consumption after the addition of FCCP and oligomycin; the maximum level of respiration was determined according to the difference in oxygen consumption after the addition of FCCP and rotenone and the respiratory capacity was determined according to the difference in oxygen consumption after the addition of FCCP and the basal level of oxygen consumption. The activity of anaerobic processes was evaluated when glucose was used as an oxidation substrate during the registration of oxygen consumption under the conditions of sequential addition of glucose, oligomycin and sodium azide to the medium. The intensity of glycolysis was determined according to the difference in oxygen consumption after adding glucose and the basal level of oxygen consumption; glycolytic capacity was determined according to the difference in oxygen consumption after adding oligomycin and glucose; and glycolytic reserve was determined according to the difference in oxygen consumption after adding glucose and sodium azide. During the analysis, the biosample volume was $275 \mu \mathrm{l}$, and $25 \mu \mathrm{l}$ of injected analyzers. Oxygen consumption was determined in ppm (17).

\section{Study of mitochondrial pore opening}

The effect of the ATACL compound and the reference drug on the opening of the mitochondrial pore was evaluated by the spectrophotometric method. The incubation medium contained: $0.5 \mathrm{ml}$ of the analyzed supernatant, $200 \mathrm{mM} \mathrm{KCl}$, and $0.5 \mathrm{ml}$ of a $1 \mu \mathrm{m}$ solution of cyclosporin A. The resulting mixture was adjusted to $2 \mathrm{ml}$ with HEPES buffer solution with a $\mathrm{pH}$ of 7.4. The optical density of the mixture was recorded at $\lambda=540 \mathrm{~nm}$, then the resulting solution was incubated for $25 \mathrm{~min}$ at room temperature with constant stirring. At the same time, the latent time of opening of the mitochondrial pore in seconds was evaluated (by changing the optical density of the incubation medium) (17).

\section{Study of mitochondrial membrane potential}

Mitochondrial membrane potential was evaluated using the spectrophotometric method. The incubation medium contained $0.5 \mathrm{ml}$ of the analyzed supernatant and $0.5 \mathrm{ml}$ of a 9 $\mu \mathrm{m}$ solution of safranin $\mathrm{O}$. The resulting mixture was adjusted to $2 \mathrm{ml}$ with HEPES buffer solution with a $\mathrm{pH}$ of 7.4. The optical density of the mixture was recorded at $\lambda=515 \mathrm{~nm}$ and $\lambda=525 \mathrm{~nm}$. The proton moving force (transmembrane electrochemical gradient, $\Delta \Psi$ ) was determined by the difference in the optical density: $\Delta \Psi=A_{515}-A_{525}$ (17).

\section{ELISA study}

In this study, the concentration of caspase- 3 was determined by enzyme-linked immunosorbent assay in the supernatant of the brain of animals. The study was performed using a specific kit for ELISA manufactured by Cloud clone corp. (USA). The biosamples preparation and the course of the analysis were consistent with the recommendations of the kit manufacturer. The results were read using an Infinite F50 microplate reader system (Tecan, Austria).

\section{Statistical analysis}

Statistical processing of the obtained data was carried out in the software package of statistical analysis STATISTICA 6.0 for Windows (StatSoft, USA). The results were expressed 
as $\mathrm{M}$ (mean) $\pm \mathrm{SEM}$. Data was checked for normal distribution (Shapiro-Wilk test). Comparison of the groups of means was performed by the method of one-way analysis of variance (ANOVA) with post-processing of Newman-Keuls (normal distribution) or Kruskal-Wallis (abnormal distribution).

\section{RESULTS}

The effect of the ATACL compound and Mexidol on the change in neurological deficit in animals suffering from cerebral ischemia

In the first step of the study, in animals of the NC group in relation to the SO group, an increase in the degree of neurological deficit by 16.4 times $(\mathrm{p}<0.01)$ was evaluated. With the prophylactic administration of the ATACL compound at doses of $50 \mathrm{mg} / \mathrm{kg}, 100 \mathrm{mg} / \mathrm{kg}, 150 \mathrm{mg} / \mathrm{kg}$, and Mexidol to animals after 24 hours of ischemia, a decrease of neurological deficit by 1.69 times compared with rabbits of the NC group by $1,69(\mathrm{p}<0.05), 2.08(\mathrm{p}<0.05), 1.63(\mathrm{p}<0.05)$ and 1.75 $(\mathrm{p}<0.05)$ times, respectively (Fig. 3$)$ was noted.

In the second step of the experiment, an increase in neurological deficit by 38.4 times $(p<0.01)$ was noted in animals of the NC group, after reproducing cerebral ischemia, in comparison with the $\mathrm{SO}$ group. At the same time, therapeutic administration (in the post ischemic period) of the ATACL compound at doses of $50 \mathrm{mg} / \mathrm{kg}, 100 \mathrm{mg} / \mathrm{kg}$ and $150 \mathrm{mg} / \mathrm{kg}$ contributed to a decrease in neurological deficit by 1.57 times $(\mathrm{p},<0.05), 2.91$ times $(\mathrm{p}<0.05)$ and 1.78 times $(\mathrm{p}<0.05)$, respectively. Moreover, the degree of neurological symptoms in animals treated with Mexidol after modeling cerebral ischemia was 1.68 times $(p<0.05)$ less than that in the $\mathrm{NC}$ group (Fig. 4). In addition, in animals that were administered the ATACL compound at a dose of $100 \mathrm{mg} / \mathrm{kg}$, neurological deficit decreased in relation to animals receiving the ATACL substance at doses of $50 \mathrm{mg} / \mathrm{kg}, 150 \mathrm{mg} / \mathrm{kg}$ and Mexidol by $84.8 \%(\mathrm{p}<0.05), 63.6 \%(\mathrm{p},<0.05)$ and $72.7 \%(\mathrm{p}<0.05)$, respectively (Fig. 3).

Figure 3. Change in the degree of neurological deficit in animals that were treated with the ATACL compound and Mexidol in conditions of cerebral ischemia

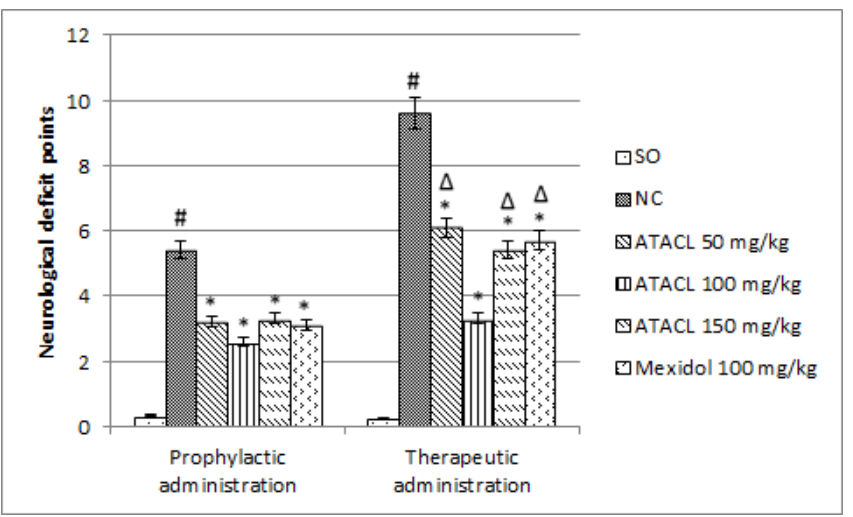

Note: \# - statistically significant relative to the SO group of animals (Newman-Keuls test $(\mathrm{p}<0.01)$ ); * - statistically significant relative to the NC group of animals (Newman-Keuls test $(\mathrm{p}<0.05)) ; \Delta$ - statistically significant relative to the group of animals treated with the ATACL compound at a dose of $100 \mathrm{mg} / \mathrm{kg}$ (Newman-Keuls test $(\mathrm{p}<0.01)$ ).

The effect of the ATACL compound and Mexidol on the change in the membrane potential of the brain mitochondria in animals under conditions of cerebral ischemia

In the first stage of the study, it was found that the value of the mitochondrial membrane potential (Fig. 5) decreased by 3.05 times $(\mathrm{p}<0.05)$ in the $\mathrm{NC}$ group of animals compared to the SO group, after 24 hours of ischemia. At the same time, in animals treated with the ATACL compound at doses of 50 $\mathrm{mg} / \mathrm{kg}, 100 \mathrm{mg} / \mathrm{kg}$ and $150 \mathrm{mg} / \mathrm{kg}$, the membrane potential of mitochondria increased in relation to the $\mathrm{NC}$ group by $58.2 \%(p<0.05), 2.17$ times $(p<0.05)$ and $80 \%(p<0.05)$, respectively. At the same time, against the background of prophylactic administration of Mexidol in rabbits, an increase in the electric potential of the mitochondrial membrane relative to the NC group of animals by $93.7 \%(\mathrm{p}<0.05)$ was noted.

In the second step of the experimental work, under conditions of therapeutic administration of the ATACL compound and Mexidol (Fig. 4), it was found that the value of the mitochondrial membrane potential in animals of the $\mathrm{NC}$ group was decreased by 9.83 times $(p<0.01)$ compared to the SO group. Moreover, against the background of the use of the compound ATACL at doses of $50 \mathrm{mg} / \mathrm{kg}, 100 \mathrm{mg} / \mathrm{kg}$ and $150 \mathrm{mg} / \mathrm{kg}$, the value of the membrane potential of the brain mitochondria increased in relation to the NC group of animals by 3.54 times $(p<0.05), 6.86$ times $(p<0.05)$ and 5.6 times $(p<0.05)$, respectively. At the same time, therapeutic administration of Mexidol to animals contributed to an increase in the mitochondrial membrane potential in comparison with the NC group by 5.7 times $(\mathrm{p}<0.05)$.

Figure 4. Change in the membrane potential of brain mitochondria in animals with the administration of the compound ATACL and Mexidol under conditions of cerebral ischemia

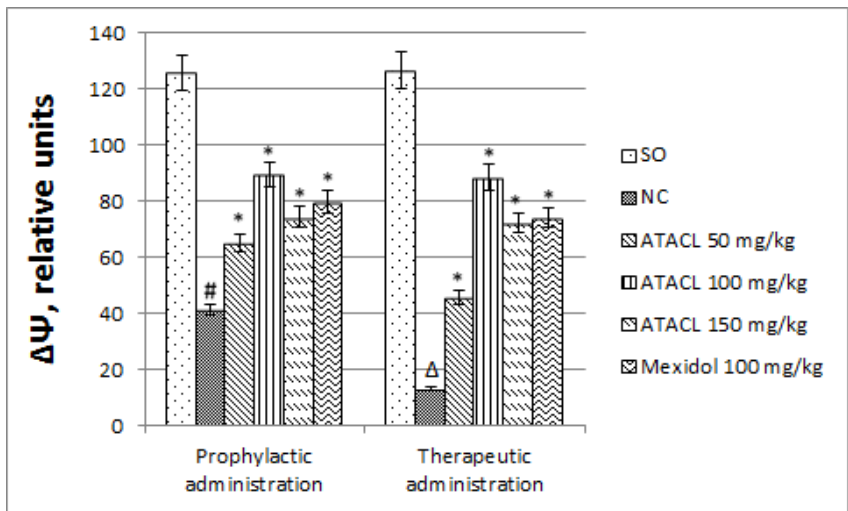


Note: statistically significant relative to the SO group of animals (Kruskal-Wallis test \# - $\mathrm{p}<0.05$; - $\mathrm{p}<0.01$ ); * - statistically significant relative to the NC group of animals (Kruskal-Wallis test $(\mathrm{p}<0.05))$.

The effect of the ATACL compound and Mexidol on the change in the degree of opening of the mitochondrial pore under cerebral ischemia

During the course of this study block, in animals of the NC group (step 1), in comparison with the SO group, a decrease of the latent time of opening of the mitochondrial pore (Fig. 5) by 3.77 times $(\mathrm{p}<0.05)$ was noted. The prophylactic administration of the ATACL compound at a dose of 50 $\mathrm{mg} / \mathrm{kg}$ did not significantly affect the degree of opening of the mitochondrial pore, while the use of this compound at doses of $100 \mathrm{mg} / \mathrm{kg}$ and $150 \mathrm{mg} / \mathrm{kg}$ contributed to an increase (relative to the $\mathrm{NC}$ group) of the latent time of mitochondrial formation pores by $90.6 \%(p<0.05)$ and $39 \%(p<0.05)$, respectively. Against the background of prophylactic administration of Mexidol, the frequency of opening of the mitochondrial pore decreased in comparison with the NC group by $51.5 \%(\mathrm{p}<0.05)$.

Under the conditions of therapeutic administration of the ATACL compound and Mexidol (step 2 of the experimental work), a decrease in the latent time of formation of the mitochondrial pore in the $\mathrm{NC}$ group of animals, with respect to the SO group, by 12.7 times $(\mathrm{p}<0.05)$ was noted. At the same time, in animals treated with the ATACL compound at doses of $50 \mathrm{mg} / \mathrm{kg} ; 100 \mathrm{mg} / \mathrm{kg}$ and $150 \mathrm{mg} / \mathrm{kg}$, the degree of opening of the mitochondrial pore was by 3.18 times $(p<0.05)$, 6.42 times $(p<0.05)$ and 5.18 times $(p<0.05)$ less than the similar value of the NC group (Fig. 5). Against the background of the use of Mexidol, an increase in the latent time of opening of the mitochondrial pore with respect to the NC group of animals by 5.3 times $(p<0.05)$ was noted. In addition, the latent time of mitochondrial pore formation in animals that were treated by ATACL compound at a dose of 100 $\mathrm{mg} / \mathrm{kg}$ was 2 times $(\mathrm{p}<0.05)$ longer than that in rabbits treated with the ATACL substance at a dose of $50 \mathrm{mg} / \mathrm{kg}$ (Fig. 5).

Figure 5. Change in latent time of opening of the mitochondrial pore in animals with the administration of the ATACL compound and Mexidol under conditions of cerebral ischemia

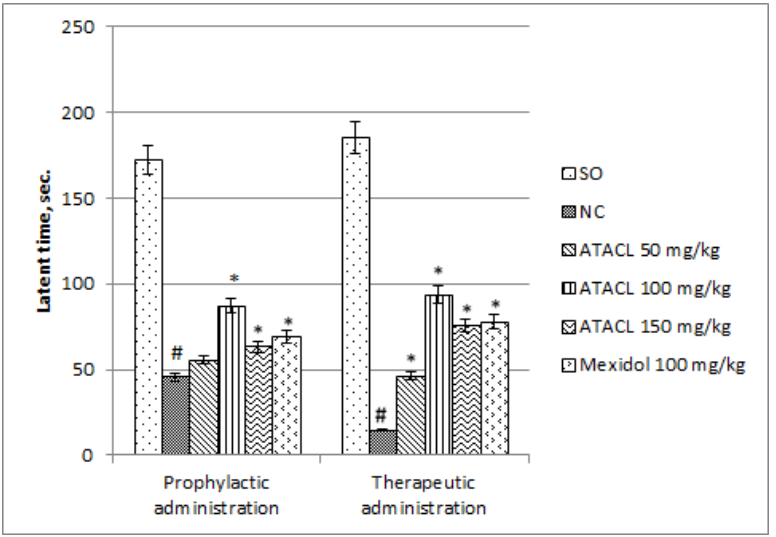

Note: \# - statistically significant relative to the SO group of animals (Newman-Keuls test $(\mathrm{p}<0.05)) ;{ }^{*}$ - statistically significant relative to the NC group of animals (Newman-Keuls test $(p<0.05)) ; \Delta$ is statistically significant relative to the group of animals treated with the ATACL compound at a dose of $100 \mathrm{mg} / \mathrm{kg}$ (Newman-Keuls test $(\mathrm{p}<0.01)$ ).

The effect of the ATACL compound and Mexidol on the change in the respirometric function of mitochondria in animals under cerebral ischemia

During the first block of the experimental study, a decrease in ATP-generating ability, maximum level of respiration and respiratory capacity in animals of the NC group, after 24-hour cerebral ischemia, relative to the SO group by 5.5 times $(p<0.01), 6$ times $(p<0.01)$ and 4.6 times $(p<0.01)$, respectively, was observed (Fig. 6). At the same time, the prophylactic use of Mexidol contributed to the increase in ATP-generating ability of animals by 3.3 times $(p<0.01)$, the maximum level of respiration by 4.1 times $(p<0.01)$ and respiratory capacity by 3.1 times $(p<0.01)$, in relation to the NC group of animals. Against the background of administration (prior to the simulation of cerebral ischemia) to animals, the ATACL compound at a dose of $50 \mathrm{mg} / \mathrm{kg}$ compared with the NC group showed an increase in ATP-generating ability, maximum respiratory rate and respiratory capacity by 2.2 times $(\mathrm{p}<0.01), 2.4$ times $(<0.01)$ and 2 times $(\mathrm{p}<0.01)$, respectively. On the other hand, when using this compound at a dose of $100 \mathrm{mg} / \mathrm{kg}$, the indicators evaluated at this stage of the experimental work increased by 4.1 times $(p<0.01), 4.8$ times $(p<0.01)$ and 4.3 times $(p<0.01)$, respectively, relative to the NC group of animals (Fig. 6). With the prophylactic use of the ATACL compound at a dose of $150 \mathrm{mg} / \mathrm{kg}$ in relation to the group of animals deprived of pharmacological support, an increase in ATP-generating ability by 3.5 times $(p<0.01)$ and the maximum level of respiration by 4.4 times $(p<0.01)$ and respiratory capacity by 3.7 times $(p<0.01)$ was observed.

Figure 6. Change in the processes of mitochondrial respiration during the prophylactic administration of the ATACL compound and Mexidol under conditions of cerebral ischemia

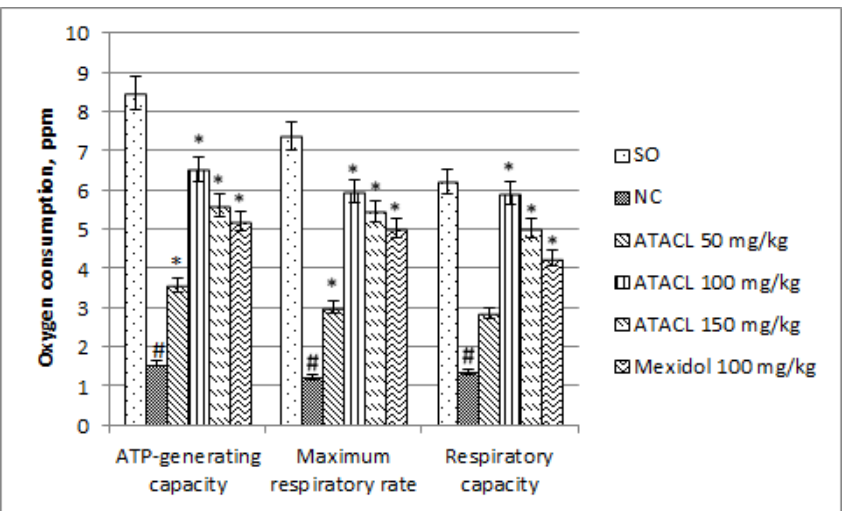

Note: \# - statistically significant relative to the SO group of animals (Kruskal-Wallis test $(\mathrm{p}<0.01)$ ); * - statistically 
significant relative to the NC group of animals (Kruskal-Wallis test $(\mathrm{p}<0.01))$.

During the second step of the study, there was a decrease in ATP-generating ability, maximum respiratory rate and respiratory capacity in animals of the NC group after 14 days of cerebral ischemia, in comparison with the SO group, by 13.3 times $(p<0.01) ; 14.5$ times $(p<0.01)$ and 9.3 times $(p<0.01)$, respectively, (Fig. 7). Against the background of therapeutic administration of Mexidol (Fig. 7) in relation to the $\mathrm{NC}$ group of animals, an increase in ATP-generating ability by 9.1 times $(p<0.01)$, maximum respiratory rate by 10.8 times $(p<0.01)$ and respiratory capacity 7.5 times $(p<0.01)$ was observed. At the same time, the therapeutic administration of the ATACL compound at a dose of $50 \mathrm{mg} / \mathrm{kg}$ contributed to the restoration of the mitochondrial respirometric function, which was expressed as an increase (in comparison with the NC group of animals) of ATP-generating ability, maximum respiratory rate and respiratory capacity by 5.2 times $(p<0.01) ; 3.7$ times $(p<0.01)$ and 3.5 times $(p<0.01)$, respectively. When using the ATACL substance at a dose of 100 $\mathrm{mg} / \mathrm{kg}$ in the post-ischemic period, an increase in ATPgenerating ability, maximum respiratory rate and respiratory capacity in relation to the group of animals lacking pharmacological support was observed 11 times $(\mathrm{p}<0.01), 12.2$ times $(p<0.01)$ and 8.6 times $(p<0.01)$, respectively. At the same time, the therapeutic administration of ATACL at a dose of $150 \mathrm{mg} / \mathrm{kg}$ contributed to an increase in ATP-generating ability relative to the $\mathrm{NC}$ of the animal group by 9.4 times $(p<0.01)$, maximum respiratory rate by 11.1 times $(p<0.01)$ and respiratory capacity by 7.5 times $(\mathrm{p}<0.01)$.

Figure 7. Change in the processes of mitochondrial respiration during therapeutic administration of the ATACL compound and Mexidol under conditions of cerebral ischemia

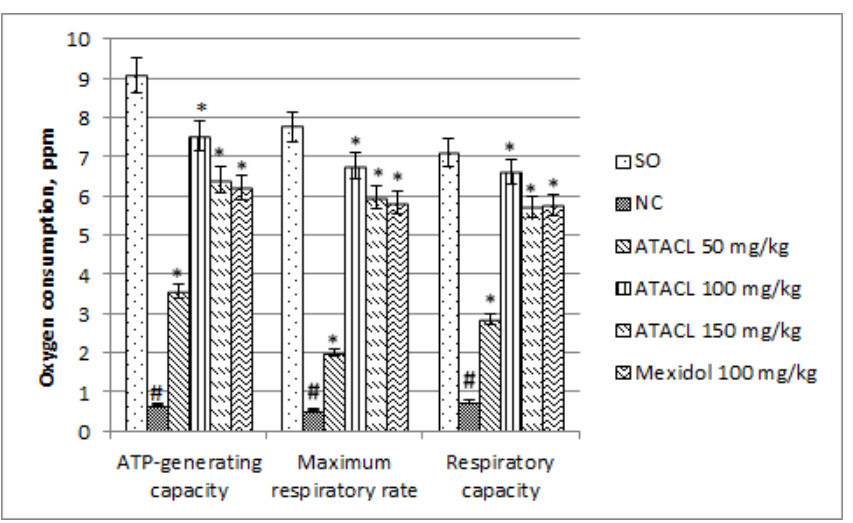

Note: \# - statistically significant relative to the SO group of animals (Kruskal-Wallis test $(\mathrm{p}<0.01)$ ); * - statistically significant relative to the NC group of animals (Kruskal-Wallis test $(\mathrm{p}<0.01))$
The effect of the ATACL compound and Mexidol on changes in the activity of anaerobic processes in animal brain tissue under cerebral ischemia

During the first step of the study, in animals of the NC group compared to the SO group, there was an increase in glycolysis intensity by 8.8 times $(p<0.001)$ and a decrease in glycolytic capacity by 4.1 times $(\mathrm{p}<0.001)$, while glycolytic reserve took a negative value (Fig. 8). Against the background of prophylactic administration of Mexidol, a restoration of the glycolytic reserve was noted, as well as an increase in glycolytic capacity by 2.2 times $(\mathrm{p}<0.001)$, accompanied by a decrease in the intensity of glycolysis compared to the NC group of animals by 2.4 times $(p<0.001)$. When using the ATACL compound at a dose of $50 \mathrm{mg} / \mathrm{kg}$, the glycolysis intensity decreased by 2.4 times $(\mathrm{p}<0.001)$ with respect to the $\mathrm{NC}$ group, while the glycolytic capacity increased by 1.5 times $(p<0.001)$ against the background of the restoration of the glycolytic reserve. At the same time, the glycolysis intensity against the background of ATACL compound administration at doses of $100 \mathrm{mg} / \mathrm{kg}$ and $150 \mathrm{mg} / \mathrm{kg}$ decreased in comparison with the NC group by 3.7 times $(\mathrm{p}<0.001)$ and 2.3 times $(p<0.001)$, respectively. The value of glycolytic capacity increased by 3.2 times $(p<0.001)$ and 1.7 times $(\mathrm{p}<0.001)$, respectively. It should be noted that in animals prophylactically treated with the ATACL compound at doses of $100 \mathrm{mg} / \mathrm{kg}$ and $150 \mathrm{mg} / \mathrm{kg}$, a restoration of the glycolytic reserve was noted (Fig. 8).

Figure 8. Change in the activity of anaerobic processes in animal brain tissue during the prophylactic administration of the ATACL compound and Mexidol under conditions of cerebral ischemia

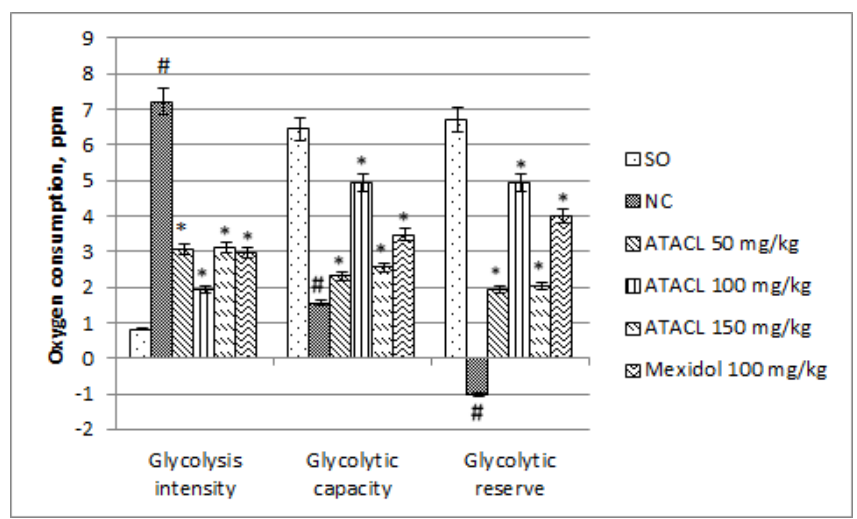

Note: \# - statistically significant relative to the SO group of animals (Newman-Keuls test $(\mathrm{p}<0.001)$ ); * - statistically significant relative to the NC group of animals (Newman-Keuls test $(\mathrm{p}<0.001))$.

At the second step of the study, in animals of the NC group after 14 days of ischemia, in comparison with the SO group, an increase in the intensity of glycolysis by 11.7 times $(p<0.001)$ was observed, accompanied by a decrease in glycolytic capacity by 6.5 times $(\mathrm{p}<0.001)$ with a negative value of the glycolytic reserve (Fig. 9). The therapeutic administration of Mexidol in relation to the $\mathrm{NC}$ group showed a 
decrease in the intensity of glycolysis by 2.9 times $(\mathrm{p}<0.001)$ and an increase in glycolytic capacity by 3.4 times $(\mathrm{p}<0.001)$ when restoring the glycolytic reserve. At the same time, against the background of the ATACL compound use at a dose of $50 \mathrm{mg} / \mathrm{kg}$, a decrease in the intensity of glycolysis and an increase in glycolytic capacity relative to animals deprived of pharmacological support by 3.4 times $(\mathrm{p}<0.001)$ and 3.7 times $(\mathrm{p}<0.001)$, respectively, were noted. In this case, the glycolysis intensity decreased by 5.4 times $(p<0.001)$ and 3 times $(p<0.001)$ when the animals were administered the ATACL compound at doses of $100 \mathrm{mg} / \mathrm{kg}$ and $150 \mathrm{mg} / \mathrm{kg}$. On the other hand, the glycolytic capacity increased when the ATACL substance was administered at doses of $100 \mathrm{mg} / \mathrm{kg}$ and $150 \mathrm{mg} / \mathrm{kg}$, compared with the NC group of animals, by 5.5 times $(\mathrm{p}<0.001)$ and 3 times (p $<0.001$ ), respectively. It should be noted that with the therapeutic use of the ATACL compound in the entire studied dose range, the restoration of the glycolytic reserve was observed (Fig. 9).

Figure 9. Changes in the activity of anaerobic processes in animal brain tissue during therapeutic administration of the

ATACL compound and Mexidol under conditions of cerebral ischemia

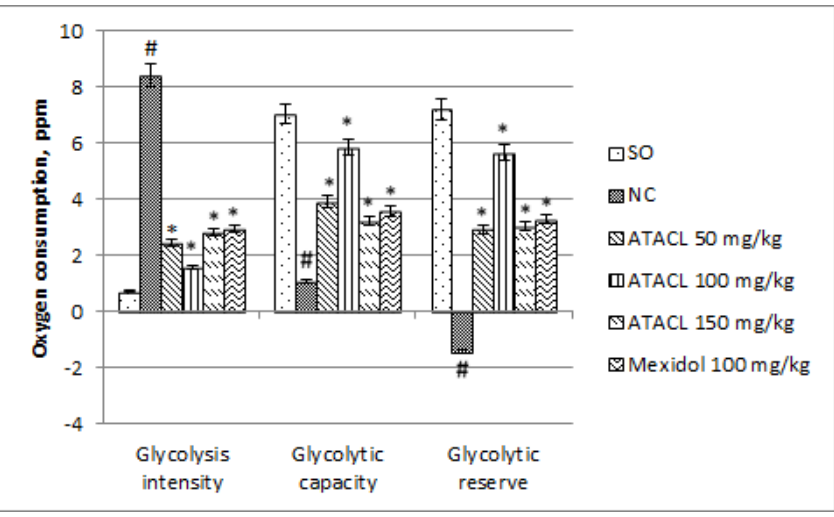

Note: \# - statistically significant relative to the SO group of animals (Newman-Keuls test $(p<0.001)$ ); * - statistically significant relative to the NC group of animals (Newman-Keuls test $(\mathrm{p}<0.001))$.

\section{The effect of the ATACL compound and Mexidol on changing the concentration of caspase -3 in animal brain tissue under cerebral ischemia}

During the first stage of the study, in animals of the NC group, in comparison with the SO group, an increase in the concentration of caspase- 3 in the brain supernatant by 10.6 times $(\mathrm{p}<0.001)$ was noted. At the same time, prophylactic administration of Mexidol contributed to a decrease in the level of capsase-3 in relation to the NK group of animals by $57.4 \%(\mathrm{p}<0.05)$. Also, the concentration of caspase- 3 decreased with the administration of $100 \mathrm{mg} / \mathrm{kg}$ and $150 \mathrm{mg} / \mathrm{kg}$ ATACL compound to animals by 2.2 times $(p<0.05)$ and $48.5 \%(p<0.05)$, while prophylactic use of this compound at a dose of $50 \mathrm{mg} / \mathrm{kg}$ did not significantly affect the change in caspase-3 level in the supernatant of animals brain (Fig. 10).
At the second stage of the study, in animals of the NC group, after 14 days of the ischemic period, the concentration of capsase-3 in the supernatant of the animals brain exceeded the similar indicator of the SO group by 12.8 times $(\mathrm{p}<0.001)$. Against the background of therapeutic administration of Mexidol in animals, a decrease in the level of caspase-3 by $2.1(\mathrm{p}<0.05)$ in comparison with the $\mathrm{NC}$ group was noted. At the same time, in animals therapeutically treated with ATACL at doses of $50 \mathrm{mg} / \mathrm{kg}, 100 \mathrm{mg} / \mathrm{kg}$ and $150 \mathrm{mg} / \mathrm{kg}$, the concentration of caspase-3 in the supernatant of the brain was lower than that in the NC group of animals by $51.5 \%(\mathrm{p}<0.05)$, by 2.6 times $(\mathrm{p}<0.05)$ and by $92.3 \%$ $(\mathrm{p}<0.05)$, respectively (Fig. 10).

Figure 10. Change in the concentration of caspase- 3 in the animals' brain tissue with the administration of the ATACL compound and Mexidol under conditions of cerebral ischemia

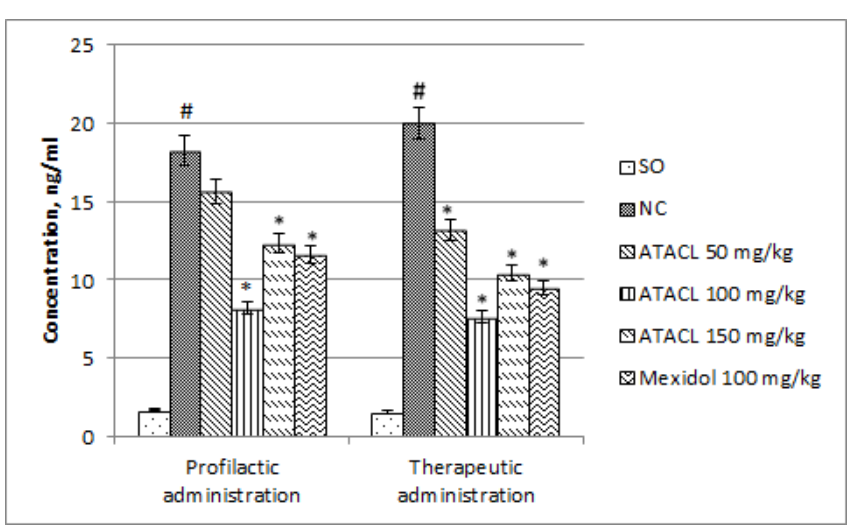

Note: \# - statistically significant relative to the SO group of animals (Newman-Keuls test $(\mathrm{p}<0.001)) ; *$ - statistically significant relative to the $\mathrm{NC}$ group of animals (Newman- Keuls test $(\mathrm{p}<0.05))$.

\section{DISCUSSION}

The high medical, social and economic impact of ischemic cerebrovascular accident makes it relevant to search for optimal treatment and prevention strategies for this condition (19). Among the existing methods of treating ischemic stroke, thrombolytic therapy that aims at fast restoration of cerebral blood flow is generally recognized (20). However, despite the proven effectiveness of thrombolytic pharmacotherapy, this pharmacological approach has significant limitations for use (such as a small therapeutic window); In addition, there is a significant risk of reperfusion complications of thrombolytic therapy (21). It is important that measures used to prevent ischemic stroke are focused on reducing the impact of risk factors (hypreglycemia, atherosclerosis, coronary heart disease) on the human body, or limited to the use of dietary supplements of cerebrotropic action, which often have little evidence of use (22). In this regard, the development of new ways of preventing and treating brain ischemic damage becomes relevant. Literary sources provide data on the positive effect of compounds - derivatives of a cinnamic acid on the functional state of brain cells 
under ischemic conditions. Thus, a study by Zhao et al, 2015 showed that the use of cinnamaldehyde helped to reduce neurological deficit and the cerebral infarction zone area, suppressed the activation of intracellular signal transduction molecules, and decreased the concentration of pro-inflammatory markers and leukocyte infiltration of the ischemic site in animals with permanent brain ischemia (23). Hemmati et.al., 2018 demonstrated the variable dose-dependent effect of cinnamic acid on the course of redox reactions and oxidative stress under conditions of streptozotocin-induced dementia. Under these experimental conditions, the use of cinnamic acid contributed to the restoration of the memorial trail in animals, normalization of the activity of antioxidant enzymes (superoxidedismutase and catalase) and a decrease in lipid peroxidation (24). Also, a study by Ren et.al.2017 showed that, in conditions of cerebral ischemia, the use of ferulic acid reduced the risk of reperfusion complications by suppressing apoptotic reactions and normalizing the antioxidant state of the cell (25). In this regard, it seems advisable to study cinnamic acid derivatives as cerebroprotective agents.

The study showed that the use of 4-hydroxy-3,5-ditertbutyl cinnamic acid helped to restore the functional activity of the rabbits brain mitochondria under conditions of cerebral ischemia. In this case, the most pronounced pharmacological effect was noted in the use of 4-hydroxy-3,5-ditertbutyl cinnamic acid at a dose of $100 \mathrm{mg} / \mathrm{kg}$. Thus, the prophylactic and therapeutic administration of this ATACL dose to animals showed a decrease in neurological deficit and contributed to a restoration of the mitochondrial membrane potential, which was higher than that in the group of animals without pharmacological support. In addition, with the prophylactic (before the modeling of ischemia) administration of 4-hydroxy-3,5-di-tertbutyl cinnamic acid, an increase in ATP-generating ability, maximal respiratory rate and respiratory capacity, respectively, was noted. Under conditions of therapeutic administration, these parameters increased as well. Also, both the prophylactic and therapeutic use of 4hydroxy-3,5-di-tertbutyl cinnamic acid contributed to the normalization of aerobic/anaerobic metabolism, and to a decrease in the concentration of the proapoptotic enzyme caspase-3.

In previous studies, the cerebroprotective properties of 4hydroxy-3,5-di-tertbutyl cinnamic acid were established (13), but the detailed mechanism of action of this compound was not established. This study represent data on the positive effect of 4-hydroxy-3,5-di-tertbutyl cinnamic acid on bioenergetic processes occurring in the brain mitochondria under cerebral ischemia, and large laboratory animals that are more susceptible to ischemic effects were used as a biological model. Thus, the mechanism of the cerebrotropic action of 4hydroxy-3,5-di-tertbutyl cinnamic acid can be mediated by the restoration of mitochondrial function. Literature also provides data on the positive effects of cinnamic acid derivatives on mitochondrial function. A study by Meeprom et.al., 2018 showed that the use of isoferulic acid reduces the degree of damage to mitochondria under conditions of methylglutoxalinduced apoptosis (26). The anti-apoptotic effect of cinnamic acid derivatives was also established by Wang et.al., 2018, where it was demonstrated that ligustrazine-cinnamon acid derivatives suppressed cytochrome $\mathrm{C}$ releasing and restored the activity of the anti-apoptotic complex Bcl-2 / Bax upon the induction of apoptosis of cobalt chloride (27). The positive effect of cinnamic acid was shown in the study by Anupama et.al., 2018, where the use of cinnamic acid contributed to the restoration of mitochondrial membrane potential and a decrease in the intensity of caspase-dependent apoptosis reactions (27). The suppression of apoptosis reactions under the influence of polyphenolic compounds, including derivatives of cinnamic acid, may be associated with the interaction of this class of compounds with components of the mitochondrial pore of transition permeability (mPTP). A study by Makoto N., et.al. 2019showed that polyphenolic compounds can directly interact with the N-terminal amino acid residues of VDAC, thereby changing the conformation of this structure and inhibiting the opening of mPTP. In addition, indirect inhibition of VDAC activity by polyphenolic compounds is possible, realized through a change in the activity of regulatory proteins - sirtuins, particulary sirtuin 1 (29). Cinnamic acid derivatives can also stimulate mitochondrial biogenesis, as confirmed by a study by Gannon et.al. 2015, in which trans-Cinnamaldehyde stimulated mitochondrial biogenesis through the activation of AMPK, PGC-1 $\alpha$, (PPARa) and PPARß / $\delta$ (29).

At the same time, 4-hydroxy-3,5-di-tertbutyl cinnamic acid compares with the favorably mitochondrial dysfunction correctors - triphenylphosphonium-based compounds, the synthesis and purification of which are laborious and not economically viable (31). At the same time, the synthesis of 4hydroxy-3,5-di-tertbutyl cinnamic acid is easily performed and does not require significant economic investments (31), which makes this compound a promising and economically affordable cerebroprotective agent with high pharmacological activity aiming at restoring mitochondrial function under conditions of cerebral ischemia.

\section{CONCLUSION}

The study showed that both prophylactic and therapeutic use of 4-hydroxy-3,5-di-tertbutyl cinnamic acid under conditions of cerebral ischemia contributed to the normalization of mitochondrial bioenergetic processes and to a decrease in caspase- 3 concentration, which ultimately contributed to a decrease in neurological symptoms in animals. Based on the obtained data, significant cerebroprotective properties of 4hydroxy-3,5-di-tertbutyl cinnamic acid, realized through the restoration of mitochondrial function, can be assumed.

\section{ETHICS APPROVAL}

All research procedures were carried out in strict accordance with the European Union Directive for the welfare of laboratory animals (No. 2010/63/EU). 


\section{COMPETING INTERESTS}

There are no conflicts of interest.

\section{FUNDING}

None.

\section{REFERENCES}

1. Feigin VL, Krishnamurthi RV, Parmar P, et al. Update on the Global Burden of Ischemic and Hemorrhagic Stroke in 1990-2013: The GBD 2013 Study. Neuroepid. 2015;45(3):161-176.

2. Ma Y, Liu Y, Zhang Z, Yang GY. Significance of Complement System in Ischemic Stroke: A Comprehensive Review. Aging Dis. 2019; 10(2): 429-462.

3. Alawieh A, Elvington A, Zhu $\mathrm{H}$, et al. Modulation of post-stroke degenerative and regenerative processes and subacute protection by site-targeted inhibition of the alternative pathway of complement. J Neuroinflammation. 2015;12:247.

4. www.who.int/news-room/fact-sheets/detail/the-top-10causes-of-death.

5. Yew WP, Djukic ND, Jayaseelan JSP, et al. Early treatment with minocycline following stroke in rats improves functional recovery and differentially modifies responses of peri-infarct microglia and astrocytes. J Neuroinflammation. 2019;16(1):6.

6. Kalogeris T, Baines CP, Krenz M, Korthuis RJ. Ischemia/Reperfusion. Compr Physiol. 2016; 7(1): 113-170.

7. Maillet A, Yadav S, Loo YL, Sachaphibulkij K, Pervaiz S. A novel Osmium-based compound targets the mitochondria and triggers ROS-dependent apoptosis in colon carcinoma. Cell Death Dis. 2013;4(6):e653.

8. Zorov DB, Juhaszova M, Sollott SJ. Mitochondrial reactive oxygen species (ROS) and ROS-induced ROS release. Physiol Rev. 2014;94(3):909-950.

9. Duan F, Yu Y, Guan R, Xu Z, Liang H, Hong L. Vitamin K2 Induces Mitochondria-Related Apoptosis in Human Bladder Cancer Cells via ROS and JNK/p38 MAPK Signal Pathways. PLoS One. 2016;11(8):e0161886.

10. Shalini S, Dorstyn L, Dawar S, Kumar S. Old, new and emerging functions of caspases. Cell Death Differ. 2015; 22(4):526-539.

11. Bano D, Prehn JHM. Apoptosis-Inducing Factor (AIF) in Physiology and Disease: The Tale of a Repented Natural Born Killer. EBioMedicine. 2018;30:29-37.

12. Pluta R, Ułamek-Kozioł M, Czuczwar SJ. Neuroprotective and Neurological/Cognitive Enhancement Effects of Curcumin after Brain Ischemia Injury with Alzheimer's Disease Phenotype. Int J Mol Sci. 2018;19(12):4002.

13. Voronkov AV, Abaev VT, Oganesyan ET, Pozdnyakov DI. Some aspects of cerebroprotective activity of 4-hydroxy-3,5-di-tretbutyl cinnamic acid in ischemic brain damage in the experiment. Med. Bull. of North Caucasus. 2018;13(1):90-93.
14. Ciprov AV, Kostina YuA. Study of cardioprotective efficacy of pyrimidine and 3-hydroxypyridine derivatives combination in anticancer chemotherapy in experiment Saratov j. med.scien.res. 2014;10(2):257-61. (in Russian)

15. McGraw KP., Pashayan AG., Wendel OT. Brain Infarction in Mongolian gerbil worsened in the treatment of phenoxybenzamine. Stroke. 1976;7(5):485- 488.

16. Patel SP, Sullivan PG, Pandya JD, et al. N-acetylcysteine amide preserves mitochondrial bioenergetics and improves functional recovery following spinal trauma. Exp Neurol. 2014;257:95-105.

17. Voronkov A.V., Pozdnyakov D.I., Nigaryan S.A., Khouri E.I., Miroshnichenko K.A., Sosnovskaya A.V., Olokhova E.A. Evaluation of the mitochondria respirometric function in the conditions of pathologies of various geneses. Pharmacy \& Pharmacology. 2019;7(1):2031.

18. Zhyliuk VI, Mamchur VV, Pavlov S. Role of functional state of neuronal mitochondria of cerebral cortex in mechanisms of nootropic activity of neuroprotectors in rats with alloxan hyperglycemia. Eksp. i klin. farm. 2015;78:10-4.

19. Chen F, Qi Z, Luo Y, et al. Non-pharmaceutical therapies for stroke: mechanisms and clinical implications. Prog Neurobiol. 2014;115:246-269.

20. Fang MC, Cutler DM, Rosen AB. Trends in thrombolytic use for ischemic stroke in the United States. J Hosp Med. 2010;5(7):406-409.

21. Lin MP, Sanossian N, Liebeskind DS. Imaging of prehospital stroke therapeutics. Expert Rev Cardiovasc Ther. 2015;13(9):1001-1015.

22. Meschia JF, Bushnell C, Boden-Albala B, et al. Guidelines for the primary prevention of stroke: a statement for healthcare professionals from the American Heart Association/American Stroke Association. Stroke. 2014; 45(12):3754-3832.

23. Zhao J, Zhang X, Dong L, et al. Cinnamaldehyde inhibits inflammation and brain damage in a mouse model of permanent cerebral ischaemia. Br J Pharmacol. 2015; 172(20):5009-5023.

24. Hemmati AA, Alboghobeish S, Ahangarpour A. Effects of cinnamic acid on memory deficits and brain oxidative stress in streptozotocin-induced diabetic mice. Korean J Physiol Pharmacol. 2018;22(3):257-267.

25. Ren Z, Zhang R, Li Y, Li Y, Yang Z, Yang H. Ferulic acid exerts neuroprotective effects against cerebral ischemia/reperfusion-induced injury via antioxidant and anti-apoptotic mechanisms in vitro and in vivo. Int $\mathrm{J}$ Mol Med. 2017;40(5):1444-1456.

26. Aramsri M, Catherine B Chan, Weerachat S, Sirichai A. Isoferulic acid attenuates methylglyoxal-induced apoptosis in INS-1 rat pancreatic $\beta$-cell through mitochondrial survival pathways and increasing glyoxalase-1 activity. Biomed. \& Pharm. 2018;101:777-85.

27. Wang P, Zhao R, Yan W, Zhang X, et.al. Neuroprotection by new ligustrazine-cinnamon acid derivatives on $\mathrm{CoCl} 2$-induced apoptosis in differentiated PC12 cells. Bioorg Chem. 2018;77:360-369. 
28. Anupama N, Preetha Rani MR, Shyni GL, Raghu KG. Glucotoxicity results in apoptosis in H9c2 cells via alteration in redox homeostasis linked mitochondrial dynamics and polyol pathway and possible reversal with cinnamic acid. Toxicol In Vitro. 2018; 53:178-192.

29. Naoi M, Wu Y, Shamoto-Nagai M, Maruyama W. Mitochondria in Neuroprotection by Phytochemicals: Bioactive Polyphenols Modulate Mitochondrial Apoptosis System, Function and Structure. Int J Mol Sci. 2019; 20(10):2451.

30. Gannon NP, Schnuck JK, Mermier CM, Conn CA, et.al. trans-Cinnamaldehyde stimulates mitochondrial biogenesis through PGC- $1 \alpha$ and PPAR $\beta / \delta$ leading to enhanced GLUT4 expression. Biochimie. 2015;119:45-51.

31. Zielonka J, Joseph J, Sikora A, et al. Mitochondria-Targeted Triphenylphosphonium-Based Compounds: Syntheses, Mechanisms of Action, and Therapeutic and Diagnostic Applications. Chem Rev. 2017;117(15):1004310120.

32. Oganesyan E.T., Shatokhin S.S., Glushko A.A. Using quantum-chemical parameters for predicting anti-radical (HO) activity of related structures containing a cinnamic mold fragment. i. derivatives of cinnamic acid, chalcon and flavanon. Pharmacy \& Pharmacology. 2019;7(1): 53-66. 